\title{
Assessing the In Vitro Drug Release from Lipid-Core Nanocapsules: a New Strategy Combining Dialysis Sac and a Continuous-Flow System
}

\author{
Diego Fontana de Andrade, ${ }_{1}^{1}$ Carine Zuglianello, ${ }^{1}$ Adriana Raffin Pohlmann, ${ }^{1,2}$ \\ Silvia Stanisçuaski Guterres, ${ }^{1}$ and Ruy Carlos Ruver Beck ${ }^{1,3}$
}

Received 11 March 2015; accepted 7 May 2015; published online 19 May 2015

\begin{abstract}
The in vitro assessment of drug release from polymeric nanocapsules suspensions is one of the most studied parameters in the development of drug-loaded nanoparticles. Nevertheless, official methods for the evaluation of drug release from submicrometric carriers are not available. In this work, a new approach to assess the in vitro drug release profile from drug-loaded lipid-core nanocapsules (LNC) was proposed. A continuous-flow system (open system) was designed to evaluate the in vitro drug release profiles from different LNC formulations containing prednisolone or clobetasol propionate (LNC-CP) as drug model (LNC-PD) using a homemade apparatus. The release medium was constantly renewed throughout the experiment. A dialysis bag containing $5 \mathrm{~mL}$ of formulation $\left(0.5 \mathrm{mg} \mathrm{mL}{ }^{-1}\right)$ was maintained inside the apparatus, under magnetic stirring and controlled temperature $\left(37^{\circ} \mathrm{C}\right)$. In parallel, studies based on the conventional dialysis sac technique (closed system) were performed. It was possible to discriminate the in vitro drug release profile of different formulations using the open system. The proposed strategy improved the sink condition, by constantly renewing the release medium, thus maintaining the drug concentration farther from the saturated concentration in the release medium. Moreover, problems due to sampling errors can be easily overcome using this semi-automated system, since the collection is done automatically without interference from the analyst. The system proposed in this paper brings important methodological and analytical advantages, becoming a promising prototype semi-automated apparatus for performing in vitro drug release studies from drug-loaded lipid-core nanocapsules and other related nanoparticle drug delivery systems.
\end{abstract}

KEY WORDS: dialysis sac technique; flow-through cell apparatus; in vitro drug release; lipid-core nanocapsules; sink condition.

\section{INTRODUCTION}

Recent scientific advances and advantages attributed to nanoparticles as drug carrier have aroused the interest of the pharmaceutical industry in these technologies. According to Bamrungsap and co-workers, 2012 (1), nanotechnology is certain to promote significant changes in the market of medicine production in the next decade. Taking into account that nanomedicines have been used in therapeutics for some time now, a consolidated regulatory system is important for the characterization of these "nanotech" products $(1,2)$.

Despite recent technological advances, analytical obstacles persist in the physicochemical characterization of

\footnotetext{
${ }^{1}$ Programa de Pós-Graduação em Ciências Farmacêuticas, Departamento de Produção e Controle de Medicamentos, Universidade Federal do Rio Grande do Sul, Avenida Ipiranga, 2952, Porto Alegre, RS 90610-000, Brazil.

${ }^{2}$ Departamento de Química Orgânica, Instituto de Química, Universidade Federal do Rio Grande do Sul, Avenida Bento Gonçalves, 9500, Porto Alegre, RS 91501-970, Brazil.

${ }^{3}$ To whom correspondence should be addressed. (e-mail: ruy.beck@ufrgs.br)
}

nanoparticles, mainly due to the colloidal nature of these systems. In this scenario, the information obtained through the combination of characterization techniques is valuable to clarify the organization of these carriers at the molecular level and predict their behavior in biological systems (3).

The in vitro assessment of drug release from polymeric nanocapsules suspensions is one of the most studied parameter in the development of drug-loaded nanoparticles formulations. Nevertheless, no official methods for the evaluation of drug release from submicrometric carriers have been developed, and no agreement has been reached among researchers about the best technique to be employed in this evaluation (4).

Besides the dissolution tests employed for conventional pharmaceutical dosage forms, the evaluation of in vitro drug release is a powerful tool in the development of new products, comprising quality control strategies for drug delivery systems and batch-to-batch control. It is also relevant as a means to support the determination of bioequivalence by in vivo methodologies (5-8).

Several in vitro methods have been used to assess the drug release from different nanoparticle formulations. According to $\mathrm{Xu}$ and co-workers, 2012 (9), the methods described can be classified into two categories: (1) sample and 
separation methods and (2) membrane diffusion methods. The disadvantages of the methods classified into the first category originate from separation processes (ultrafiltration, ultracentrifugation, or centrifugal ultrafiltration techniques) that should be used to separate the drug already released and the portion that remains associated with nanocarriers. However, the application of centrifugal forces at the nanostructurated systems may destabilize them, leading to premature drug release (10). Furthermore, drug losses may occur during the sampling process, causing errors in in vitro drug release profiles $(6,9)$. Among the methods that belong to the second category are the dialysis sac $(3,11)$, reverse dialysis sac $(4,9)$, and microdialysis techniques (12). The use of small pore size membranes in these techniques solves one of the major limitations of other in vitro drug release techniques: the separation of the drug already released from the drug-loaded nanocarriers from the remaining encapsulated drug (4).

The conventional method of dialysis sac has been severely criticized for not providing the sink condition inside the bag, reducing the ability of the predictive power of in vitro-in vivo correlation to some routes of administration. Nevertheless, this method is able to mimic the physiological condition of dosage forms that remain immobilized after administration, as observed using intramuscular or subcutaneous routes $(4,8)$.

In recent years, some studies have proposed the use of different USP dissolution apparatus to assess the drug release profile from micro- or nanostructured systems such as apparatus I, II, and IV, showing their advantages and disadvantages (6,7,13-15). Among the studies performed, the flow-through cell apparatus seems to be the best alternative to conduct in vitro release studies on dispersed systems due to its high discriminative power and to the fact that it can easily be converted in an automated process $(7,13,15)$. Nevertheless, it is known that the polymer matrix that composes the polymeric nanoparticles may clog the membrane used in these techniques, causing variations in flow throughout the experiment (8). In addition, methods based on high amounts of release medium may affect the analytical phase, requiring high concentration of the sample to prevent loss of analytical sensitivity.

In this scenario, considering the advantages of the dialysis sac and of the flow-through cell methods, and aiming to overcome their disadvantages, this paper proposed a novel semiautomated method to assess the in vitro drug release profile from lipid-core nanocapsule suspensions. A system that combines the separation of the released drug from that still associated with lipid-core nanocapsules (dialysis membrane) and a device coupled to a peristaltic pump that allows a continuous flow of the release medium allowing the maintenance of sink condition was developed. This system was tested to discriminate the diffusion profile of clobetasol propionate and prednisolone in solution from their release from lipid-core nanocapsules. The choice of these model drugs was based on the different drug distribution in the nanocarriers, according to a prediction based on the drug $\log \mathrm{D}$ values, as proposed by Oliveira and co-workers (2013) (16). According to that study, prednisolone (PD) is partially dissolved in water and partially dispersed in the colloid, mainly in the polymeric wall of the lipid-core nanocapsules, while clobetasol propionate $(\mathrm{CP})$ is fully incorporated into the lipid core of these colloidal structures and protected by their polymeric wall.

\section{MATERIALS AND METHODS}

\section{Materials}

CP was donated by Neo Química (Goiás, Brazil). PD, Poly( $\varepsilon$-caprolactone) (PCL), sorbitan monostearate, and dialysis tubing cellulose membrane (molecular weight cutoff $12 \mathrm{kDa}$, avg. flat width $25 \mathrm{~mm}$ ) were purchased from SigmaAldrich (São Paulo, Brazil). The caprylic/capric triglyceride was supplied by Brasquim (Porto Alegre, Brazil). Polysorbate 80 was acquired from Henryfarma (São Paulo, Brazil). Ethanol and acetone were obtained from Nuclear (São Paulo, Brazil). Polyethylene glycol 400 USP (PEG 400) was acquired from Synth (São Paulo, Brazil). Potassium phosphate monobasic USP and sodium hydroxide PA were obtained from VETEC (Rio de Janeiro, Brazil). HPLC grade methanol and acetonitrile were purchased from Tedia (São Paulo, Brazil). All other reagents employed in the experiments were of analytical grade and used as received.

\section{Preparation of Lipid-Core Nanocapsules}

Lipid-core nanocapsules (LNC) were prepared by interfacial deposition of a preformed polymer at a drug concentration of $0.5 \mathrm{mg} \mathrm{mL}^{-1}(17,18)$. Poly( $\varepsilon$-caprolactone) $80,000 \mathrm{MW}$ $(0.25 \mathrm{~g})$, caprylic/capric triglyceride $(412 \mu \mathrm{L})$, sorbitan monostearate $(0.095 \mathrm{~g})$, and drug (PD or CP) (0.0125 g) were dissolved at $40^{\circ} \mathrm{C}$ in $68 \mathrm{~mL}$ of acetone. This organic solution was injected under moderate magnetic stirring into an aqueous solution containing polysorbate $80(0.1925 \mathrm{~g})$. The acetone was immediately removed and the aqueous phase concentrated by evaporation (bath at $40^{\circ} \mathrm{C}$ ) under reduced pressure. Finally, the volume of the formulation $(25 \mathrm{~mL})$ was adjusted. The formulation containing PD was called LNC-PD and that containing $\mathrm{CP}$ was called LNC-CP. Each formulation was prepared in triplicate and stored in amber glass bottle at room temperature $\left(22 \pm 2^{\circ} \mathrm{C}\right)$.

\section{Characterization of Lipid-Core Nanocapsules}

\section{Particle Size Analysis, Polydispersity Index, and Zeta Potential}

To rule out the presence of micrometric populations of particles in LNC formulations, laser diffraction analyses were carried out (Mastersizer ${ }^{\circledR}$ 2000, Malvern Instruments, Malvern, UK) pouring each sample directly into the wet unit until a minimal obscuration of $2 \%$ was reached. Particle size and polydispersity index were determined at $25^{\circ} \mathrm{C}$ by photon correlation spectroscopy (Zetasizer Nano ZS®, Malvern Instruments, Malvern, UK) after previous dilution of the LNC suspensions with ultrapure water $(1: 500 \mathrm{v} / \mathrm{v})$. Zeta potentials were estimated based on electrophoretic mobility measured using the same instrument at $25^{\circ} \mathrm{C}$, after the dilution of the samples in $10 \mathrm{mM} \mathrm{NaCl}$ aqueous solution (1:500v/v). All analyses were performed in triplicate.

\section{Determination of Drug Content, Encapsulation Efficiency, and $\mathrm{pH}$}

PD was assayed by reversed phase high performance liquid chromatography (RP-HPLC) using a method reported 
by Katzer et al., 2014 (19). The system consisted of a Shimadzu HPLC (LC-20A Prominence, Shimadzu, Japan) and a Gemini RP-18 column $\left(150 \mathrm{~mm} \times 4.60 \mathrm{~mm}, 5 \mu \mathrm{m}\right.$, Phenomenex ${ }^{\circledR}$, Torrance, USA). PD was eluted using a mobile phase consisting of acetonitrile:water $(40: 60 \mathrm{v} / \mathrm{v})$ at the flow rate of

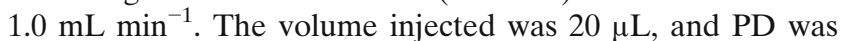
detected at $241 \mathrm{~nm}$. The validated parameter showed linearity between 10.0 and $30.0 \mu \mathrm{g} \mathrm{mL}^{-1}(y=46407 x+2918.2, r=0.9999)$ and inter-day and intra-day variability lower than $2.7 \%$. Specificity was tested in the presence of the adjuvants of lipid-core nanocapsules and demonstrated that they did not alter the PD assay. Total drug content was determined after dissolving LNC-PD $(400 \mu \mathrm{L})$ in $10 \mathrm{~mL}$ of acetonitrile. Free PD (non-associated to lipid-core nanocapsules) was determined in the ultrafiltrate after separation of the nanostructures by ultrafiltration/centrifugation technique (Ultrafree Microcon, 10,000 MW, Millipore®, Darmstadt, Germany) at $386 \mathrm{~g}$ for $10 \mathrm{~min}$. The ultrafiltate was diluted in acetonitrile $(1: 20 \mathrm{v} / \mathrm{v})$ and assayed by the RP-HPLC method previously described to determine the amount of free PD.

CP was also assayed by RP-HPLC using a method reported by Fontana et al., 2011 (11). The system consisted of a Shimadzu HPLC (LC-20A Prominence, Shimadzu, Japan) and a Gemini RP-18 column $(250 \mathrm{~mm} \times 4.60 \mathrm{~mm}, 5 \mu \mathrm{m}$, Phenomenex ${ }^{\circledR}$, Torrance, USA). CP was eluted using a mobile phase consisting of methanol:water $(80: 20 \mathrm{v} / \mathrm{v})$ at the flow rate of $1.0 \mathrm{~mL} \mathrm{~min}^{-1}$. The volume injected was $20 \mu \mathrm{L}$ and $\mathrm{CP}$ was detected at $241 \mathrm{~nm}$ (11). The validated parameter showed linearity between 10.0 and $30.0 \mu \mathrm{g} \mathrm{mL}^{-1}(y=40329 x-19,236$, $r=0.9996)$ and inter-day and intra-day variability lower than $2.8 \%$. Specificity was tested in the presence of the adjuvants of lipid-core nanocapsules and demonstrated that these factors did not alter the CP assay. Total drug content was determined after dissolution of LNC-CP $(400 \mu \mathrm{L})$ in $10 \mathrm{~mL}$ of acetonitrile. Free CP (non-associated to lipid-core nanocapsules) was determined in the ultrafiltrate after separation of the nanostructures by ultrafiltration/centrifugation technique (Ultrafree Microcon, 10.000 MWCO, Millipore ${ }^{\circ}$, Darmstadt, Germany) at $8600 \mathrm{~g}$ for $10 \mathrm{~min}$. The ultrafiltrate was not diluted and was quantified by the RP-HPLC method previously described to determine free clobetasol. In both cases, encapsulation efficiency (\%) was calculated by the difference between the total and free drug concentrations. $\mathrm{pH}$ values were determined directly in the samples using a calibrated potentiometer (VB-10, Denver Instrument, Arvada, USA). Drug content, encapsulation efficiency, and $\mathrm{pH}$ were determined in triplicate.

\section{Solubility Studies}

The saturation concentrations of PD in phosphate buffered saline (PBS) $\mathrm{pH} 7.4$ and of $\mathrm{CP}$ in water/polysorbate 80 / PEG $400(60: 0.5: 40 v / v)(11)$ were determined as follows: an excess of drug (PD or $\mathrm{CP}$ ) was added to reaction tube containing a fixed volume $(2 \mathrm{~mL})$ of the release medium (PBS or water/polysorbate $80 / \mathrm{PEG} 400$, respectively). The mixture was stirred for $1 \mathrm{~min}$ and then allowed to stand by $24 \mathrm{~h}$ at room temperature $\left(22 \pm 2^{\circ} \mathrm{C}\right)$, protected from light. The samples were centrifuged at $8600 \mathrm{~g}$ for $10 \mathrm{~min}$. An aliquot of supernatant was collected and filtered through a $0.22-\mu \mathrm{m}$ PVDF membrane (Millipore®, Darmstadt, Germany). The filtrate was diluted with fresh release media (1:100), and the concentrations of PD or CP were measured by RP-HPLC according to the methods previously described.

\section{Distribution Coefficients}

To determine the PD and CP $\log \mathrm{D}$, the software ACDLogD 6.0 (Advanced Chemistry Development, Inc., Toronto, Canada) was used. The experimental $\mathrm{pH}$ values of LNC formulations determined as described above were used to estimate these coefficients.

\section{In Vitro Drug Release Studies}

\section{Conventional Dialysis Sac Method}

The in vitro drug diffusion profiles of non-encapsulated PD or CP [hydroethanolic $(1: 10 v / v)$ or ethanolic solutions, respectively] and the release profiles of drug-loaded lipid-core nanocapsules (LNC-PD or LNC-CP) were evaluated. The dialysis bag $(12 \mathrm{kDa})$ (surface area $=17.58 \pm 1.15 \mathrm{~cm}^{2}$ ) containing $5 \mathrm{~mL}$ of non-encapsulated drug solution or LNC suspension $\left(0.5 \mathrm{mg} \mathrm{mL}^{-1}\right)$ were put in a $250-\mathrm{mL}$ beaker containing $200 \mathrm{~mL}$ of release medium (PBS pH 7.4 for PD and water/polysorbate 80/PEG 400 (60:40:0.5v/v) for CP formulations). The systems were maintained at $37^{\circ} \mathrm{C}$ with constant magnetic stirring (400 and 300 RPM for PD and CP formulations, respectively) during the experiment. All experiments were performed in triplicate, and each replicate was analyzed independently on different days. At a predetermined time interval, $2 \mathrm{~mL}$ of the external medium were withdrawn from the beaker middle position $(5 \mathrm{~cm}$ of height from the bottom of the beaker) and replaced with fresh medium to ensure the sink condition. The samples were filtered through a $0.45-\mu \mathrm{m}$ PVDF membrane (Millipore ${ }^{\circledR}$, Darmstadt, Germany), and the drug concentration of PD or CP were determined by RP-HPLC according to the methods described before, with slight modifications, in order to improve the quantification limit. In both $\mathrm{PD}$ and $\mathrm{CP}$ assays, the injection volume was adjusted to $100 \mu \mathrm{L}$ and the analytical range was from 0.5 to $15 \mu \mathrm{g} \mathrm{mL}^{-1}$ and from 0.5 to $10 \mu \mathrm{g} \mathrm{mL}^{-1}$ for PD and $\mathrm{CP}$ quantification, respectively. Taking into account these slight changes (range and injection volume), the RP-HPLC method to assay PD showed the following validation features: specificity, linearity $(y=234,458 x+54,255, r=0.9990)$, range $\left(0.5-15 \mu \mathrm{g} \mathrm{mL}{ }^{-1}\right)$, precision (repeatability and intermediate precision lower than $2.7 \%$ ), detection, and quantification

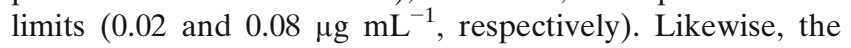
RP-HPLC method to assay CP showed the following validation data: specificity, linearity $(y=100,813 x+7505, r=0.9990)$, range $\left(0.5-10 \mu \mathrm{g} \mathrm{mL} \mathrm{m}^{-1}\right)$, precision (repeatability and intermediate precision lower than $4.1 \%$ ), detection, and quantification limits ( 0.05 and $0.19 \mu \mathrm{g} \mathrm{mL}^{-1}$, respectively).

In order to evaluate the influence of the withdrawal position on the beaker, another independent experiment was performed in a similar way. The in vitro $\mathrm{CP}$ diffusion profile from ethanolic solution was evaluated. However, in this experiment, the samples $(1 \mathrm{~mL})$ were collected at predetermined time intervals and at different heights of the release medium (beaker): at low $(2 \mathrm{~cm})$, middle $(5 \mathrm{~cm})$, and upper $(8 \mathrm{~cm})$ positions. The total height of the release medium into the 
beaker was $10 \mathrm{~cm}$. The same method described in this item was applied to assay the $\mathrm{CP}$ concentration in the samples. This study also was performed in triplicate. To enable the comparison of the different in vitro drug release profiles, the steadystate flux was calculated by fitting the profiles to the Fick's first law $(20,21)$.

\section{Proposed Release Apparatus Method (Continuous-Flow Apparatus)}

In the same way, the in vitro drug diffusion profiles of non-encapsulated PD or CP [hydroethanolic $(1: 10 v / v)$ or ethanolic solutions, respectively] and the release of the drugloaded lipid-core nanocapsules (LNC-PD or LNC-CP) were evaluated. The dialysis bag $(12 \mathrm{kDa})$ (surface area $=16.95 \pm$ $0.90 \mathrm{~cm}^{2}$ ) containing $5 \mathrm{~mL}$ of non-encapsulated drug solution or LNC suspension $\left(0.5 \mathrm{mg} \mathrm{mL}^{-1}\right)$ were put in the proposed release device (Fig. 1) containing $220 \mathrm{~mL}$ of release medium (PBS pH 7.4 for PD or water/polysorbate 80/PEG 400 (40:60:0.5) for CP formulations). The designed system was closed with a glass lid. Then, two PVC pump tubing (GILSON®, Bedfordshire, UK) were connected to the release medium input and output with the aid of rubber seals.

The free end of the pump tubing inlet was completely immersed in a beaker containing fresh release medium. Otherwise, the free end of pump tubing outlet was put in a sampling flask (Fig. 2). It is important to note that both PVC pump tubing were connected independently in the peristaltic pump. However, both were subjected to the same influence of the pump, producing the same inflow and outflow of the release medium into/out the system.

The peristaltic pump (GILSON $®$, Bedfordshire, UK) was started, and samples were constantly collected. The peristaltic pump was previously calibrated, and the flow was adjusted to $0.5 \mathrm{~mL} \mathrm{~min}^{-1}$ for formulations containing PD and $0.2 \mathrm{~mL} \mathrm{~min}^{-1}$

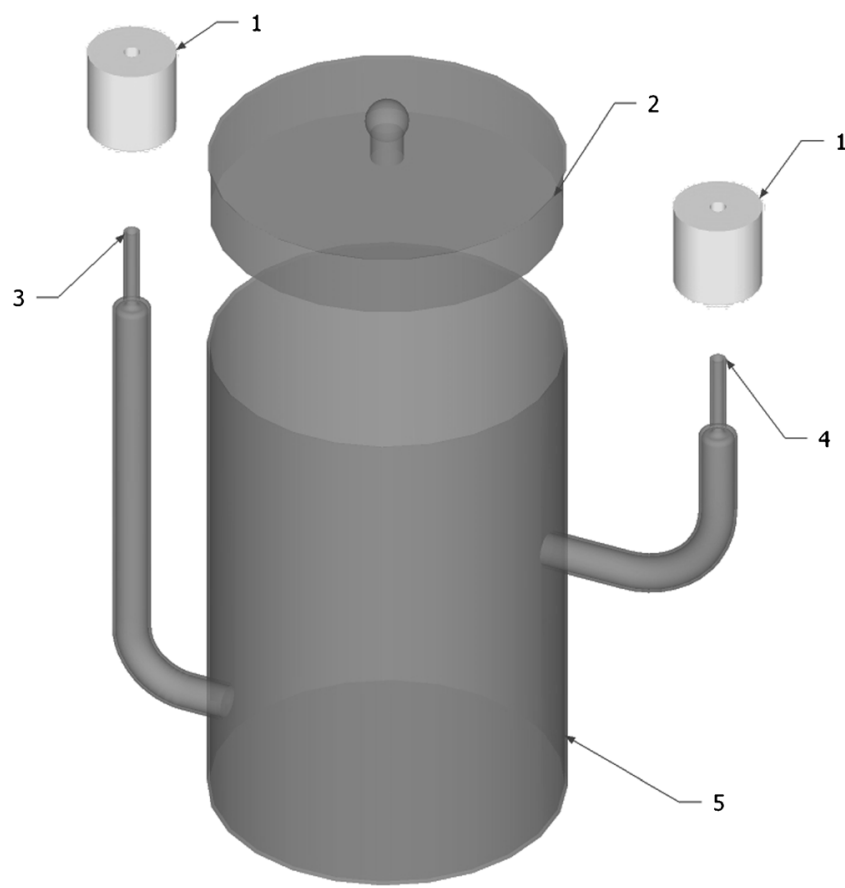

Fig. 1. Design of the proposed release device: 1 rubber seals, 2 glass lid, 3 release medium outlet, 4 release medium inlet, and 5 release device for formulations containing CP. To achieve these flows, PVC pump tubing measuring 0.76 and $0.38 \mathrm{~mm}$ in internal diameter were used. The systems were maintained at $37 \pm 2^{\circ} \mathrm{C}$ (the proposed release device (Fig. 1) was kept in a heating bath throughout the experiment) with constant magnetic stirring (400 and 300 RPM to PD and CP formulations, respectively) during the experiment. At predetermined time intervals, the samples were filtered through a $0.45-\mu \mathrm{m}$ PVDF membrane (Millipore ${ }^{\circledR}$, Darmstadt, Germany) and the drug (PD or CP) was assayed by RP-HPLC according to the methods described before. All experiments were performed in triplicate, and each replicate was analyzed independently on different days. As done for the data obtained using the conventional dialysis technique, the steady-state flux of the different in vitro drug release profiles obtained by the proposed release method was calculated by fitting the profiles to the Fick's first law.

\section{Statistical Analysis}

All formulations were prepared and analyzed in triplicate. Results are expressed as mean \pm SD (standard deviation). Student's $t$ test was applied to compare the results obtained from in vitro drug release studies. Statistical significance was considered when $p \leq 0.05$. Data analyses were performed with GraphPad Prism® software, version 5.01.

\section{RESULTS}

All drug-loaded lipid-core nanocapsules (LNC-PD and LNC-CP) had macroscopic homogeneous appearance, like a milky bluish opalescent liquid after preparation, common feature of similar LNC suspensions reported previously (11). Laser diffraction analyses afforded to exclude the presence of micrometric populations of particles in both LNC formulations, since only a monomodal nanometric population was observed for LNC-PD (D[4.3]=176 $\pm 4 \mathrm{~nm}, \operatorname{Span}=1.34 \pm 0.01)$ and LNC-CP $(\mathrm{D}[4.3]=195 \pm 5 \mathrm{~nm}, \mathrm{Span}=1.39 \pm 0.01)$.

The physicochemical characteristics of the LNC formulations are presented in Table I. LNC-PD and LNC-CP presented similar mean diameters $(184-197 \mathrm{~nm})$, acid $\mathrm{pH}$ values (5.92-6.34), negative zeta potentials (between -5.70 and $-10.81 \mathrm{mV}$ ), and polydispersity index below 0.1 , suggesting their homogeneity.

LNC suspensions containing the different drugs (PD or CP) presented drug content similar to the expected value $\left(0.5 \mathrm{mg} \mathrm{mL}^{-1}\right)$. However, the encapsulation efficiency was dependent on the drug present in each formulation. Lower values were observed for LNC-PD $(27 \pm 8.0 \%)$ than for LNC-CP $(100 \pm 0.1 \%)(p>0.05)$. The $\log \mathrm{D}$ values calculated for PD and CP were 1.49 and 3.98, respectively. Furthermore, the PD solubility in PBS pH 7.4 and CP solubility in water/polysorbate $80 / \mathrm{PEG} 400$ (40:60:0.5) were 0.34 and $0.15 \mathrm{mg} / \mathrm{mL}$, respectively.

Figure 3 shows the PD in vitro diffusion/release profiles of hydroethanolic and LNC-PD formulations obtained using the conventional dialysis sac technique and the proposed continuous-flow apparatus.

In a similar way, Fig. 4 shows the $\mathrm{CP}$ in vitro diffusion/ release profiles from ethanolic and LNC-CP formulations, respectively, obtained using the conventional dialysis sac technique and the continuous-flow apparatus. 


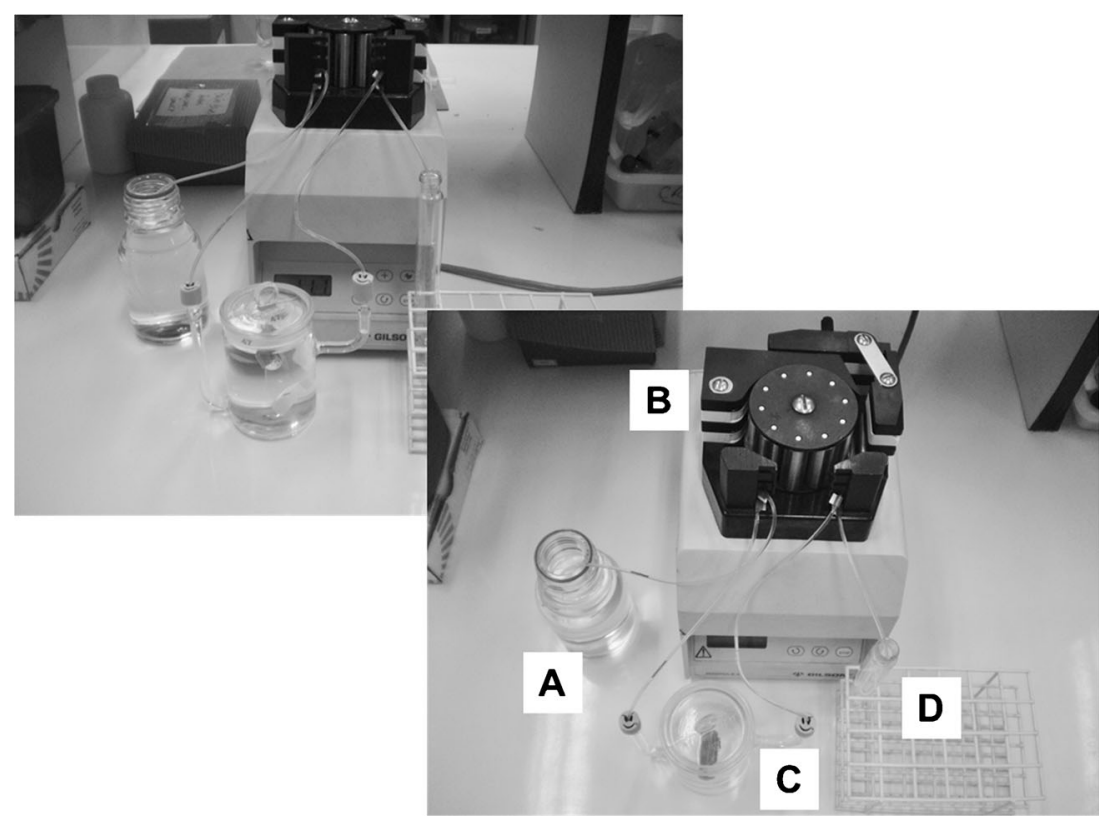

Fig. 2. Schematics of the proposed system (continuous-flow apparatus) for in vitro drug release studies composed by a flask containing fresh release medium, $\mathbf{b}$ a peristaltic pump, c the proposed release device, and $\mathbf{d}$ a sampling flask

In order to compare the different in vitro drug diffusion/ release profiles, the steady-state flux was calculated by fitting all profiles to the Fick's first law. The flux values obtained for PD hydroethanolic solution, CP ethanolic solution, LNC-PD, and LNC-CP are shown in Table II.

It is important to highlight that the comparison about the same assay performed with both approaches (conventional dialysis sac and continuous-flow system) for all formulations (PD, CP, LNC-PD, and LNC-CP) was carried out and no statistical significances were observed $(p>0.05)$.

The conventional (dialysis sac) and the proposed systems (continuous-flow apparatus) were evaluated for their ability to maintain the drug concentration in the release medium far from the drug saturation concentration in this medium. The comparison was carried out by the evaluation of the index $\mathrm{M}$, obtained by the ratio between the arithmetic mean of the drug concentration measured in the samples throughout the experiment and drug saturation concentration in the release medium $(M)$, according to Eq. (1).

$M=\frac{1}{N \cdot C_{\mathrm{sat}}} \sum_{n=1}^{N} C_{n}, n=1,2, \ldots, N$

where $\mathrm{N}$ is the total number of samples, $C_{n}$ is the drug concentration in the sample at time $t_{n}$, and $C_{\text {sat }}$ means the drug saturation concentration in the release medium. The results are shown in Fig. 5.
Another important parameter highlighted in this study was the precision of the data generated by the different in vitro drug release methods. It is noted that the standard deviation of the data is smaller in the profiles constructed from the data obtained by the in vitro drug release studies from different LNC formulations (Figs. $3 b$ and $4 b$ ), when the proposed system is used to conduct these experiments. This observation can be clearly seen concerning the formulation containing clobetasol propionate (LNC-CP).

Moreover, the influence of the withdrawal position on the beaker (higher, medium, and lower sites) in the experiment performed with the $\mathrm{CP}$ ethanolic solution $(n=3)$ is shown in Fig. 6.

As shown in Fig. 6, the in vitro drug release profile is quite different when the sampling is carried out in the upper site of the beaker compared to the profiles obtained from the middle and lower sites. This is clearly observed analyzing the data of the steady-state fluxes $(J)$ obtained by fitting the in vitro $\mathrm{CP}$ release profiles from ethanolic solutions obtained using the conventional dialysis sac technique, whose samples were collected from different sites (upper, middle, and lower medium), to the Fick's first law (Table III).

The steady-state flux $(J)$ obtained by modeling the data generated from the sampling on the upper medium is around 10 times higher $(p<0.5)$ than the values obtained by modeling data generated from sampling in the middle and lower medium.

Table I. Physicochemical Characteristics of Prednisolone-Loaded Lipid-Core Nanocapsules (LNC-PD) and Clobetasol Propionate Lipid-Core Nanocapsules (LNC-CP)

\begin{tabular}{|c|c|c|c|c|c|c|}
\hline Formulation & Drug content (mg mL ${ }^{-1}$ ) & E.E. $(\%)$ & $\mathrm{pH}$ & Particle size (nm) & PDI & Zeta potential $(\mathrm{mV})$ \\
\hline LNC-PD & $0.50 \pm 0.01$ & $27 \pm 8.0$ & $6.29 \pm 0.05$ & $189 \pm 5$ & $0.08 \pm 0.01$ & $-8.95 \pm 1.86$ \\
\hline LNC-CP & $0.50 \pm 0.03$ & $100 \pm 0.1$ & $6.09 \pm 0.17$ & $196 \pm 1$ & $0.09 \pm 0.01$ & $-5.91 \pm 0.21$ \\
\hline
\end{tabular}




\section{DISCUSSION}

The drug-loaded lipid-core nanocapsules (LNC-PD and LNC-CP) showed monomodal distribution in the nanometric range, without particle populations in the micrometric scale. All formulations had the expected drug content. Particle size, zeta potential, and $\mathrm{pH}$ were in agreement with previous studies using corticosteroids-loaded lipid-core nanocapsules $(11,22,23)$.

The difference in the encapsulation efficiency between the two different drug-loaded lipid-core nanocapsules may be associated with the different physicochemical characteristics of these drugs (PD and CP), such as lipophilicity, illustrated by their different calculated $\log \mathrm{D}$ values. According to Stella and co-workers (24), drug encapsulation efficiency for colloidal suspensions increases with the lipophilicity of the drug. Oliveira and co-workers (16) established that the $\log \mathrm{D}$ value is the best physicochemical parameter to predict the type of drug distribution in lipid-core nanocapsules. Taking into account the correlation previously established and the calculated $\log \mathrm{D}$ values, PD partly dissolves in water. The undissolved part is dispersed in the colloid, preferably in the
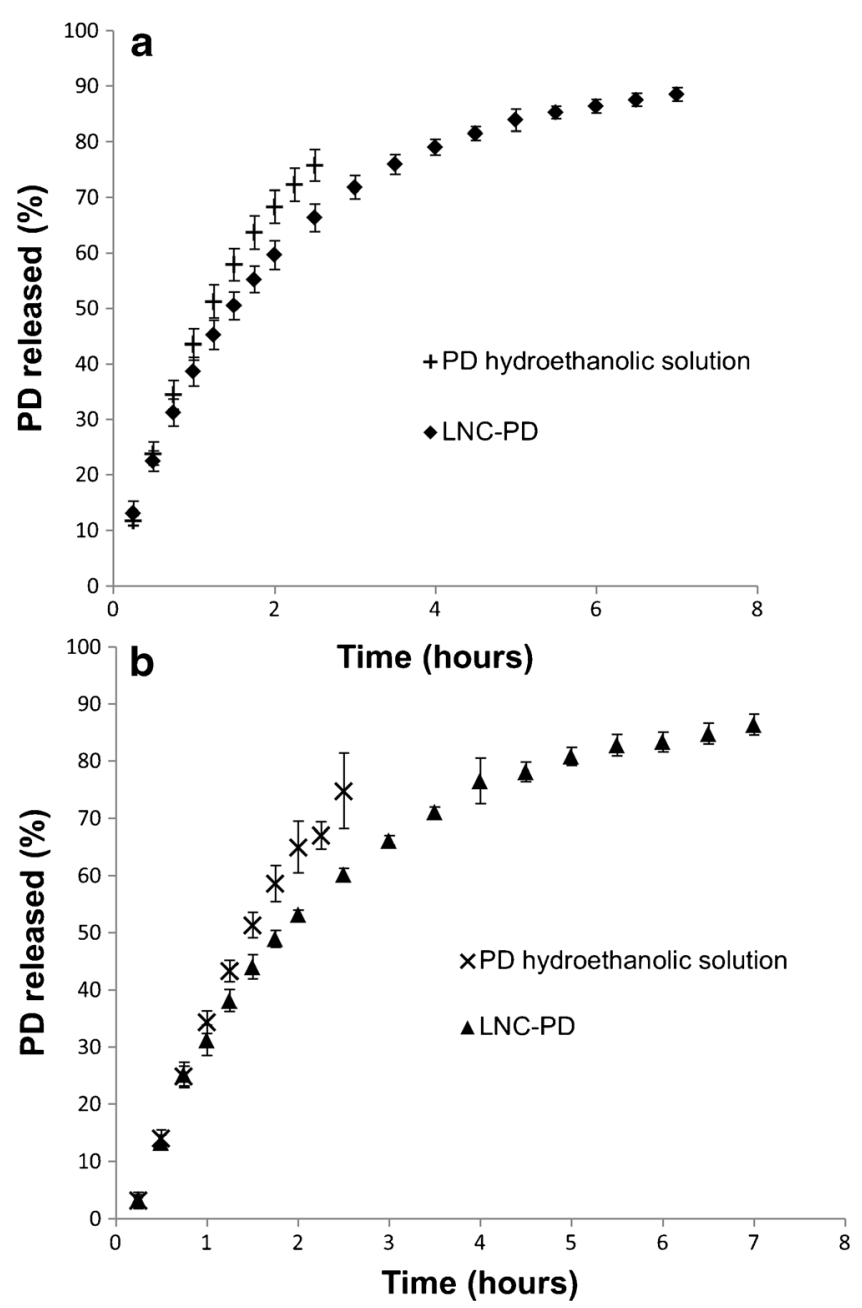

Fig. 3. In vitro $\mathrm{PD}$ diffusion/release profiles from hydroethanolic solution and lipid-core nanocapsules (LNC-PD) using a the conventional dialysis sac technique and the $\mathbf{b}$ proposed continuous-flow apparatus $(n=3)$ polymeric wall of the lipid-core nanocapsules. In turn, $\mathrm{CP}$ is fully included into the lipid core of these colloidal structures.

This prediction above becomes more reliable when the in vitro drug release profiles are considered, in which about $90 \%$ of PD was released from lipid-core nanocapsules in $5 \mathrm{~h}$, while in the same timeframe, the fraction of clobetasol released from the nanocarriers was less than $4 \%$, regardless of the in vitro drug release method. In this context, it is important to highlight that it was possible to build an in vitro drug release profile from different formulations using the continuous-flow method proposed in this study. Furthermore, the continuousflow apparatus enables the discrimination between the drug profiles differently distributed in the nanostructured formulation. The method allows obtaining discriminating profiles between samples containing the encapsulated drug from those containing its free form, even if low percentage of the drug is associated with the carriers, as in the case of prednisoloneloaded lipid-core nanocapsules (LNC-PD). In agreement with our results, Bhardwaj and Burgess (7) demonstrated the feasibility of adapting the USP apparatus IV combined with dialysis membranes for evaluating the in vitro release profile of dexamethasone-loaded liposomes. The system proposed by
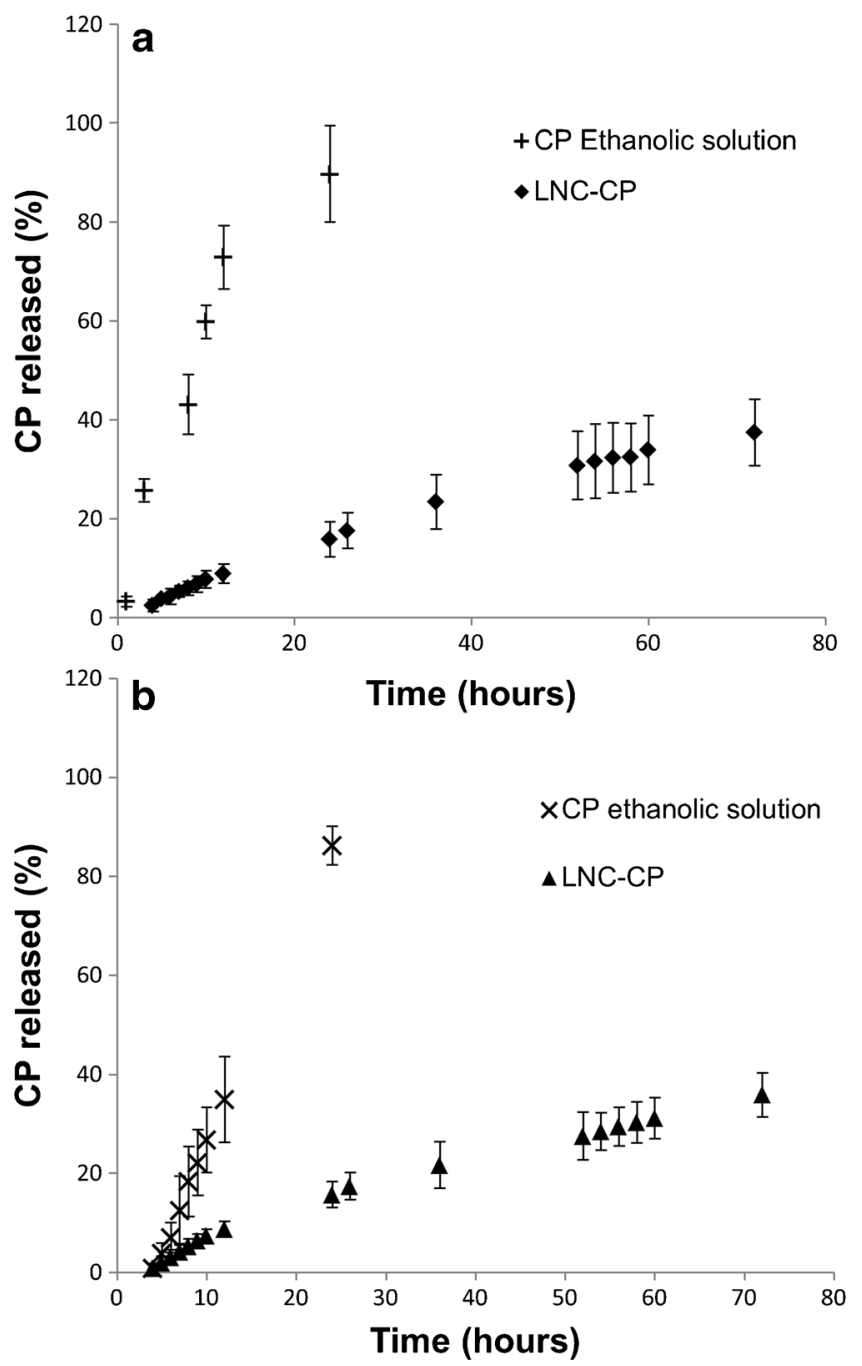

Fig. 4. In vitro $\mathrm{CP}$ diffusion/release profiles from ethanolic solution and lipid-core nanocapsules (LNC-CP) using a the conventional dialysis sac technique and the $\mathbf{b}$ continuous-flow apparatus $(n=3)$ 
Table II. Steady-State Fluxes $(J)$ Calculated by Fitting the In Vitro PD and CP Diffusion/Release Profiles Obtained Using the Conventional Dialysis Sac Technique and the Continuous-Flow Apparatus to the Fick's First Law

\begin{tabular}{lcccc}
\hline & \multicolumn{2}{c}{ Dialysis sac method } & \multicolumn{2}{c}{ Continuous-flow apparatus } \\
\cline { 2 - 4 } & $J\left(\mu \mathrm{g} \mathrm{cm}^{-2} \mathrm{~h}^{-1}\right)$ & $r$ & $J\left(\mu \mathrm{g} \mathrm{cm}^{-2} \mathrm{~h}^{-1}\right)$ \\
\hline PD solution & $47.60 \pm 4.00$ & $0.98 \pm 0.01$ & $55.54 \pm 7.42$ & \\
LNC-PD & $37.24 \pm 4.31^{a}$ & $0.98 \pm 0.01$ & $42.69 \pm 2.96^{a}$ & $0.99 \pm 0.01$ \\
CP solution & $8.20 \pm 0.68$ & $0.96 \pm 0.02$ & $0.30 \pm 1.77$ & $0.99 \pm 0.01$ \\
LNC-CP & $0.78 \pm 0.10^{b}$ & $0.99 \pm 0.01$ & $0.76 \pm 0.04^{b}$ & $0.99 \pm 0.01$ \\
\hline
\end{tabular}

${ }^{a}$ Means, in column, statistically different values compared to PD hydroethanolic solution $(p \leq 0.05)$

${ }^{b}$ Means, in column, statistically different values compared to CP ethanolic solution $(p \leq 0.05)$

the authors had more discriminating power between different formulations than between the dialysis sac and reverse dialysis techniques.

It is well established that maintaining the sink condition is a crucial requirement for in vitro drug release studies in the

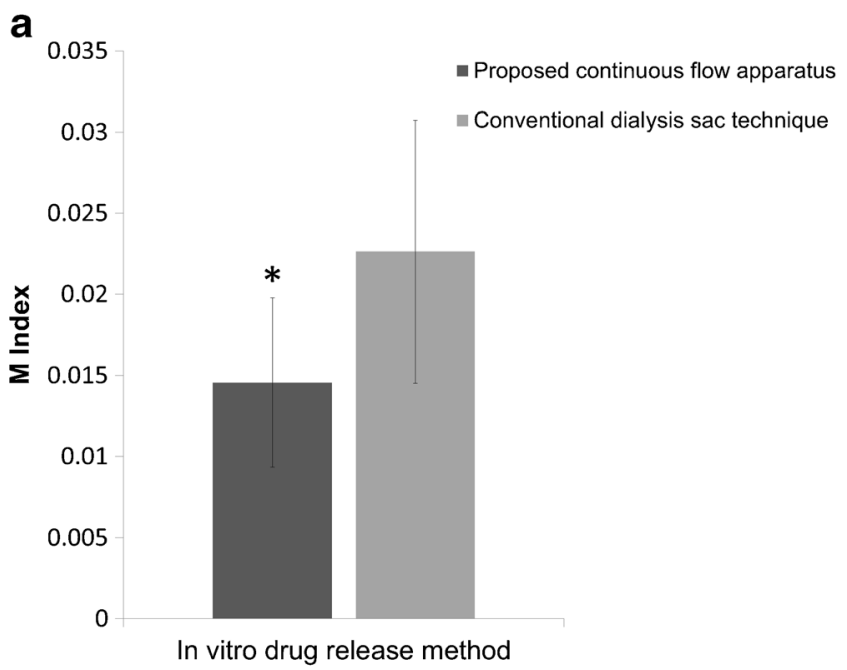

b

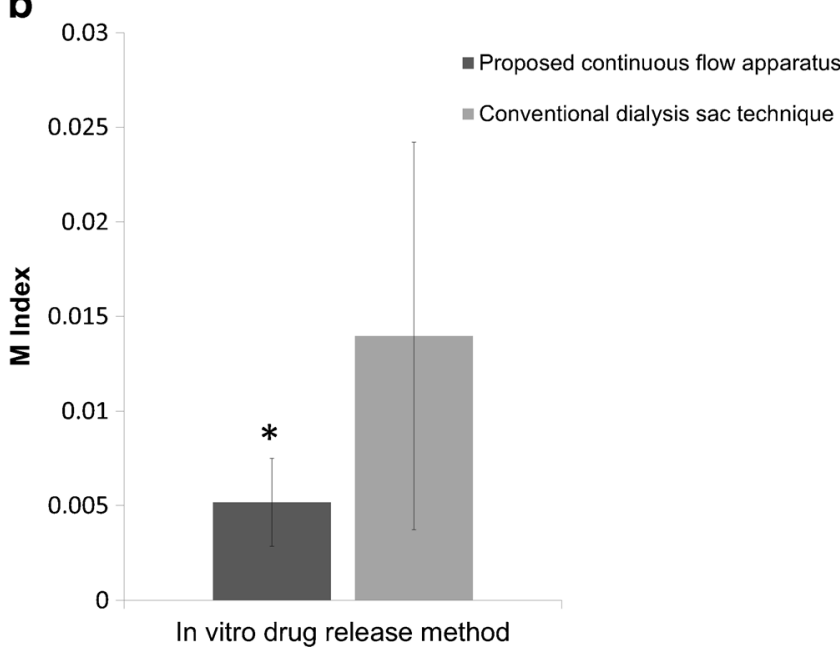

Fig. 5. Ratio between arithmetic mean of the drug concentration measured in the samples throughout the experiment and drug saturation concentration in the release medium ( $M$ index) employing the different methods (conventional dialysis sac technique and continuous-flow apparatus). a Values obtained by the in vitro PD release from LNC-PD studies. b Values obtained by the in vitro CP release from LNC-CP studies. *Statistically different values $(p \leq 0.05)$ pharmaceutical field $(25,26)$. The sink condition means that drug concentration in the dissolution or release medium may not exceed 10 to $33 \%$ of drug solubility in these fluids $(27,28)$. Aiming to maintain this condition to assess the in vitro drug release profiles, several approaches have been adopted, like the use of surfactants, solvents, and co-solvents in release medium as well as the use of the USP apparatus IV (26).

The $M$ index was calculated by the ratio between the arithmetic mean of the drug concentration measured in the samples throughout the experiment and the drug saturation concentration in the release medium for both release systems. Smaller ratio values mean lower concentration of drug in the medium. Considering the calculated values, the continuousflow apparatus proposed in this study is a viable alternative for the maintenance of sink condition for in vitro drug release studies from lipid-core nanocapsules. It ensures a higher difference from the drug concentration into the release medium to the drug saturation concentration in this one. The amount of drug assayed in all points of the experiment was 2-3 times lower in the continuous-flow method than in the conventional dialysis sac technique.

Moreover, no differences in the drug release profiles from the diverse formulations were observed, regardless the type of system (continuous-flow or closed system), as could be observed by the similar steady-state fluxes comparing these two methods, regardless the type of encapsulated drug (PD or $\mathrm{CP}$ ). This result can be explained by the maintaining of the sink conditions during all the release experiments, regardless the technique used. However, it can be clearly observed that the proposed continuous-flow system contributed to reduce the drug concentration in the release medium, without adversely affecting the drug assay in the experiments, due to its lower concentration.

Although the release apparatus proposed in this work has some advantages previously reported for continuous-flow systems (like USP apparatus IV), in this new concept, the release medium does not have to pass through a membrane. The formulations are contained in dialysis bags inside the release apparatus device. This strategy allows overcoming an important issue observed in similar flow-through cell systems, such as the flow obstruction by the particles clogging the membrane $(7,26)$.

One of the imperative parameters to validate a dissolution test is the precision of the data generated (29). According to the International Conference on Harmonisation of Technical Requirements for Registration of Pharmaceuticals for Human Use (ICH) (30), precision expresses the closeness of agreement between a series of measurements obtained 


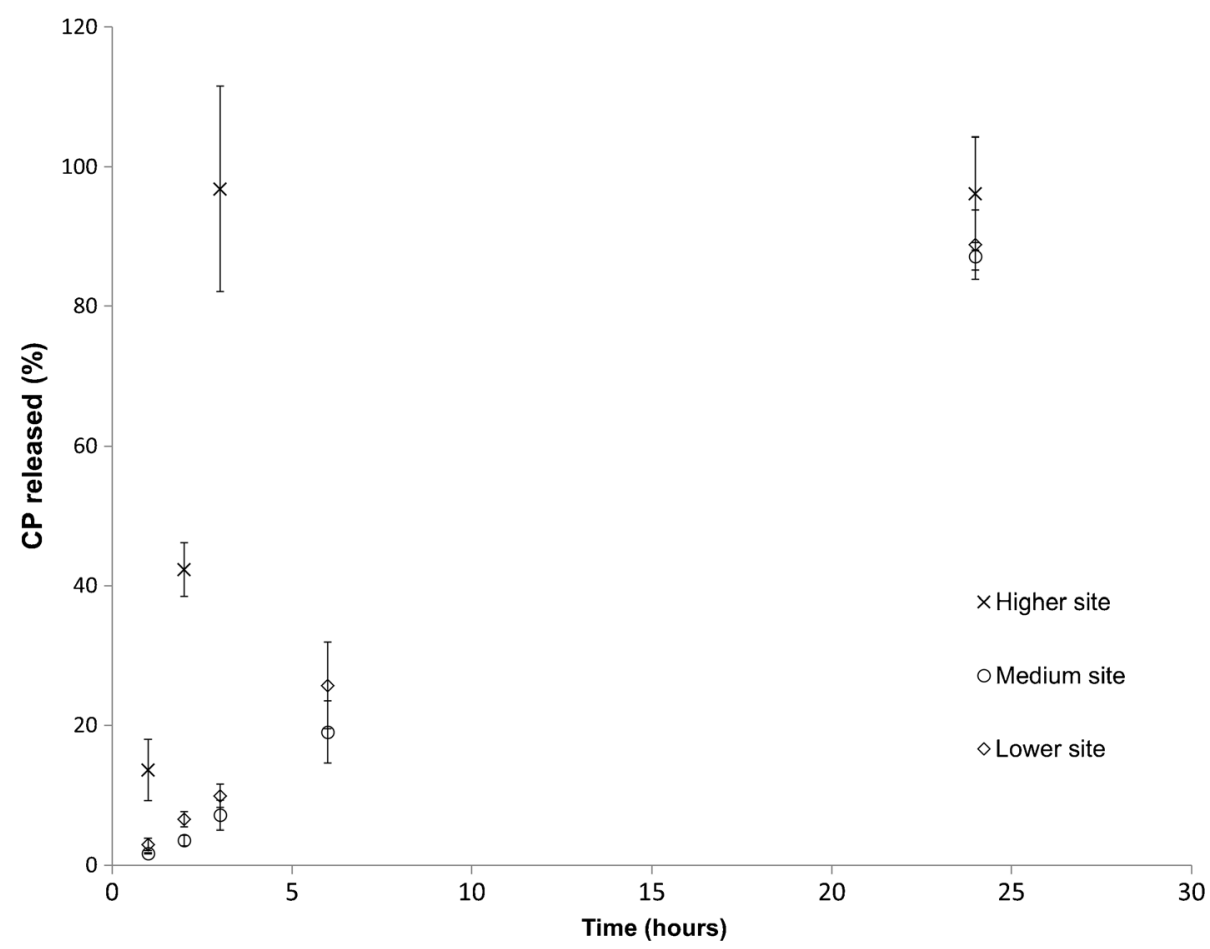

Fig. 6. In vitro $\mathrm{CP}$ release profiles from ethanolic solution using the conventional dialysis sac technique when the samples were obtained from different sampling sites (higher, medium, and lower sites). *Statistically different values $(p \leq 0.05)$

from multiple sampling of same homogeneous sample under the prescribed conditions and is usually expressed as the variance, standard deviation, or coefficient of variation of a series of measurements. However, some previous studies reported high standard deviation values (represented in graphs points of drug release profiles) for in vitro drug release studies from nanostructured systems using the dialysis sac method (11) or different USP apparatus such as the apparatus IV $(7,13)$. This way, taking into account the observed tendency for lower standard deviation in the drug release profiles achieving using the continuous-flow method compared to the conventional dialysis method, we may suggest that the continuous-flow apparatus proposed in this study is able to provide better data precision, compared to conventional dialysis sac method.

Finally, the in vitro drug release profiles from lipid-core nanocapsules were quite different when the sampling was held in the upper site of the beaker (upper medium) compared to the profiles obtained from the middle and lower medium. The influence of the analyst (experience and meticulousness to take the samples) could be clearly evidenced in the calculated steady-state fluxes values. However, the contribution of the high viscosity of the release medium (water/polysorbate
80/PEG 400-60:0.5:40v/v) to the largest standard deviation observed and the variation of the profiles obtained from different sampling sites cannot be ruled out, for the experiment conducted with the formulation LNC-CP. Therefore, these results show that, using the semi-automated continuous-flow system proposed in this paper, this limitation can be easily overcome, since sampling is carried out automatically at the same site without the interference of the analyst.

\section{CONCLUSION}

The data generated by the continuous-flow system proposed in this study as a tool to evaluate drug release from lipid-core nanocapsules are precise and adequate and allow discriminating the release from encapsulated and nonencapsulated drug. Its suitability was demonstrated either graphically as well as by comparison between the steadystate fluxes of PD or CP from nanocapsule suspensions. The proposed system is suggested as a potential alternative to maintain the sink condition during drug release studies from nanocarriers, as the release medium is continuously renewed. Therefore, it can be considered a promising semi-automated

Table III. Steady-State Fluxes $(J)$ Obtained by Fitting the In Vitro CP Diffusion Profiles from Ethanolic Solutions $(N=3)$ Using the Conventional Dialysis Sac Technique and Samples Collected from Different Sites (Upper, Middle, and Lower Medium) to the Fick’s First Law

\begin{tabular}{|c|c|c|c|c|c|c|}
\hline & \multicolumn{2}{|l|}{ Upper medium } & \multicolumn{2}{|l|}{ Middle medium } & \multicolumn{2}{|l|}{ Lower medium } \\
\hline & $J\left(\mu \mathrm{g} \mathrm{cm}^{-2} \mathrm{~h}^{1}\right)$ & $r$ & $J\left(\mu \mathrm{g} \mathrm{cm}^{-2} \mathrm{~h}^{-1}\right)$ & $r$ & $J\left(\mu \mathrm{g} \mathrm{cm}^{-2} \mathrm{~h}^{-1}\right)$ & $r$ \\
\hline $\mathrm{CP}$ solution & $52.25 \pm 5.49^{\mathrm{a}}$ & $0.97 \pm 0.01$ & $4.79 \pm 0.88^{\mathrm{b}}$ & $0.99 \pm 0.01$ & $4.73 \pm 0.77^{b}$ & $0.99 \pm 0.01$ \\
\hline
\end{tabular}


prototype apparatus for performing in vitro drug release studies from nanocarriers.

\section{ACKNOWLEDGMENTS}

D. F. de Andrade thanks CAPES-Brazil for his Master Fellowship. The authors gratefully acknowledge Ricardo Samuel Citolin for his generous contribution to improve the paper quality. The authors thank the financial support of CAPES, CNPq, FAPERGS, and INCT-CNPq/MCTI/Brazil.

\section{REFERENCES}

1. Bamrungsap $\mathrm{S}$, Zhao Z, Chen $\mathrm{T}$, Wang L, Li C, Fu T, et al. Nanotechnology in therapeutics: a focus on nanoparticles as a drug delivery system. Nanomedicine. 2012;7(8):1253-71. doi:10.2217/nnm.12.87.

2. Mangematin V, Walsh S. The future of nanotechnologies. Technovation. 2012;32:157-60. doi:10.1016/j.technovation. 2012.01.003.

3. Schaffazick SR, Guterres SS, Freitas LL, Pohlmann AR. Caracterização e estabilidade físico-química de sistemas poliméricos para a administração de fármacos. Quim Nova. 2003;26(5):726-37. doi:10.1590/S0100-40422003000500017.

4. Ferronato K, Bruxel F, Araújo FA, Teixeira HF, Koester LS. Emprego do método da bolsa de diálise da avaliação da liberação de fármacos a partir de emulsões submicrométricas. Lat Am J Pharm. 2010;29(2):313-20.

5. Sinko PJ. Físico-Farmácia e Ciências Farmacêuticas. 5th ed. Porto Alegre: Artmed; 2008.

6. Abdel-Mottaleb MM, Lamprecht A. Standardized in vitro drug release test for colloidal drug carriers using modified USP dissolution apparatus I. Drug Dev Ind Pharm. 2011;37(2):178-84. doi:10.3109/03639045.2010.502534.

7. Bhardwaj U, Burgess DJ. A novel USP apparatus 4 based release testing method for dispersed systems. Int J Pharm. 2010;388:28794. doi:10.1016/j.ijpharm.2010.01.009.

8. D'Souza SS, DeLuca PP. Methods to assess in vitro drug release from injectable polymeric particulate systems. Pharm Res. 2006;23(3):460-74.

9. Xu X, Khan MA, Burgess DJ. A two-stage reverse dialysis in vitro dissolution testing method for passive targeted liposomes. Int J Pharm. 2012;426:211-8. doi:10.1016/j.ijpharm.2012.01.030.

10. Moreno-Bautista G, Tam KC. Evaluation of dialysis membrane process for quantifying the in vitro drug-release from colloidal drug carriers. Colloids Surf A Physicochem Eng Asp. 2011;389:299-303. doi:10.1016/j.colsurfa.2011.07.032.

11. Fontana MC, Coradini K, Guterres SS, Pohlmann AR, Beck RC. Nanoencapsulation as a way to control the release and to increase the photostability of clobetasol propionate: influence of the nanostructured system. J Biomed Nanotechnol. 2009;5(3):254-63.

12. Hitzman CJ, Wiedmann TS, Dai H, Elmquist WF. Measurement of drug release from microcarriers by microdialysis. J Pharm Sci. 2005;94(7):1456-66. doi:10.1002/jps.20349.

13. Sievens-Figueroa L, Pandya N, Bhakay A, Keyvan G, MichniakKohn B, Bilgili E, et al. Using USP I and USP IV for discriminating dissolution rates of nano- and microparticle-loaded pharmaceutical strip-films. AAPS PharmSciTech. 2012;13(4):1473-82. doi:10.1208/s12249-012-9875-3.

14. Esposito E, Mazzitelli S, Cortesi R, Drechsler M, Ravani L, Nastruzzi C. Analysis of the drug release profiles from formulations based on micro and nano systems. Curr Anal Chem. 2013:9:37-46. doi:10.2174/1573411011309010037.

15. Heng D, Cutler DJ, Chan HK, Yun J, Raper JA. What is a suitable dissolution method for drug nanoparticles? Pharm Res. 2008;25(7):1696-701. doi:10.1007/s11095-008-9560-0.

16. Oliveira CP, Venturini CG, Donida B, Poletto FS, Guterres SS, Pohlmann AR. An algorithm to determine the mechanism of drug distribution in lipid-core nanocapsule formulations. Soft Matter. 2013;9:1141-50. doi:10.1039/C2SM26959G.

17. Venturini CG, Jäger E, Oliveira CP, Bernardi A, Battastini AM, Guterres SS, et al. Formulation of lipid-core nanocapsules. Colloids Surf A Physicochem Eng Asp. 2011;375:200-8. doi:10.1016/ j.colsurfa.2010.12.011.

18. Jäger E, Venturini CG, Poletto FS, Colomé LM, Pohlmann JP, Bernardi A, et al. Sustained release from lipid-core nanocapsules by varying the core viscosity and the particle surface area. J Biomed Nanotechnol. 2009;5(1):130-40. doi:10.1166/ jbn.2009.1004.

19. Katzer T, Chaves P, Bernadi A, Pohlmann AR, Guterres SS, Beck RCR. Prednisolone-loaded nanocapsules as ocular drug delivery system: development, in vitro drug release and eye toxicity. J Microencapsul. 2014;31(6):519-28. doi:10.3109/ 02652048.2013.879930.

20. Poletto FS, Jäger E, Cruz L, Pohlmann AR, Guterres SS. The effect of polymeric wall on the permeability of drug-loaded nanocapsules. Mater Sci Eng C Mater Biol Appl. 2008;28(4):472-8. doi:10.1016/j.msec.2007.04.015.

21. Contri RV, Kaiser M, Poletto FS, Pohlmann AR, Guterres SS. Simultaneous control of capsaicinoids release from polymeric nanocapsules. J Nanosci Nanotechnol. 2011;11:2398-406. doi:10.1166/jnn.2011.3521.

22. Marchiori ML, Lubini G, Dalla Nora G, Friedrich RB, Fontana $\mathrm{MC}$, Ourique AF, et al. Hydrogel containing dexamethasoneloaded nanocapsules for cutaneous administration: preparation, characterization, and in vitro drug release study. Drug Dev Ind Pharm. 2010;36(8):962-71. doi:10.3109/03639041003598960.

23. Fontana MC, Rezer JF, Coradini K, Leal DB, Beck RC. Improved efficacy in the treatment of contact dermatitis in rats by a dermatological nanomedicine containing clobetasol propionate. Eur J Pharm Biopharm. 2011;79(2):241-9. doi:10.1016/ j.ejpb.2011.05.002.

24. Stella B, Arpicco S, Rocco F, Marsaud V, Renoir J, Cattel L, et al. Encapsulation of gemcitabine lipophilic derivatives into polycyanoacrylate nanospheres and nanocapsules. Int J Pharm. 2007;344:71-7. doi:10.1016/j.ijpharm.2007.06.006.

25. Dokoumetzidis A, Macheras P. A century of dissolution research: from Noyes and Whitney to the biopharmaceutics classification system. Int J Pharm. 2006;321:1-11. doi:10.1016/ j.ijpharm.2006.07.011.

26. Phillips DJ, Pygall SR, Cooper VB, Mann JC. Overcoming sink limitations in dissolution testing: a review of traditional methods and the potential utility of biphasic systems. J Pharm Pharmacol. 2012;64(11):1549-59. doi:10.1111/j.2042-7158.2012.01523.x.

27. US Pharmacopeial Forum. Pharmacopeial previews. 2004;30:35163.

28. Rossi RC, Dias CL, Donato EM, Martins LA, Bergold AM, Fröehlich PE. Development and validation of dissolution test for ritonavir soft gelatin capsules based on in vivo data. Int $\mathrm{J}$ Pharm. 2007;338:119-24. doi:10.1016/j.ijpharm.2007.01.036.

29. U.S. Departmant of Healt and Human Services, Food and Drug Administration, Center for Drug Evaluation and Research (CDER). Dissolution testing of immediate release solid oral dosage forms. Washington, DC: U.S. Government Printing Office; 1997.

30. International Conference of Harmonization (ICH). Validation of analytical procedures, text and methodology Q2(R1); 2005. 